Research Article

\title{
On the total chromatic edge stability number and the total chromatic subdivision number of graphs
}

\author{
Arnfried Kemnitz*, Massimiliano Marangio \\ Computational Mathematics, Technical University Braunschweig, Universitätsplatz 2, 38106 Braunschweig, Germany
}

(Received: 13 October 2021. Received in revised form: 9 February 2022. Accepted: 15 February 2022. Published online: 22 February 2022.$)$

(c) 2022 the authors. This is an open access article under the CC BY (International 4.0) license (www.creativecommons.org/licenses/by/4.0/).

\begin{abstract}
A proper total coloring of a graph $G$ is an assignment of colors to the vertices and edges of $G$ (together called the elements of $G$ ) such that neighbored elements-two adjacent vertices or two adjacent edges or a vertex and an incident edge-are colored differently. The total chromatic number $\chi^{\prime \prime}(G)$ of $G$ is defined as the minimum number of colors in a proper total coloring of $G$. In this paper, we study the stability of the total chromatic number of a graph with respect to two operations, namely removing edges and subdividing edges, which leads to the following two invariants. (i) The total chromatic edge stability number or $\chi^{\prime \prime}$-edge stability number $e s_{\chi^{\prime \prime}}(G)$ is the minimum number of edges of $G$ whose removal results in a graph $H \subseteq G$ with $\chi^{\prime \prime}(H) \neq \chi^{\prime \prime}(G)$ or with $E(H)=\emptyset$. (ii) The total chromatic subdivision number or $\chi^{\prime \prime}$-subdivision number $s d_{\chi^{\prime \prime}}(G)$ is the minimum number of edges of $G$ whose subdivision results in a graph $H \subseteq G$ with $\chi^{\prime \prime}(H) \neq \chi^{\prime \prime}(G)$ or with $E(H)=\emptyset$. We prove general lower and upper bounds for $e s_{\chi^{\prime \prime}}(G)$. Moreover, we determine $e s_{\chi^{\prime \prime}}(G)$ and $s d_{\chi^{\prime \prime}}(G)$ for some classes of graphs.
\end{abstract}

Keywords: total chromatic edge stability number; total chromatic subdivision number; total chromatic number; total coloring.

2020 Mathematics Subject Classification: 05C15.

\section{Introduction}

We consider finite simple graphs $G=\left(V(G), E(G)\right.$ ). A (graph) invariant $\rho(G)$ is a function $\rho: \mathcal{I} \rightarrow \mathbb{R}_{0}^{+} \cup\{\infty\}$ where $\mathcal{I}$ is the class of finite simple graphs and $\mathbb{R}_{0}^{+}$the set of non-negative real numbers. Invariants mostly are integer-valued. An empty graph is a graph with empty edge set.

It is an interesting topic to determine the stability of an arbitrary invariant $\rho(G)$ of a graph $G$ with respect to two operations, namely removing edges and subdividing edges. The $\rho$-edge stability number and the $\rho$-subdivision number of $G$ are defined as follows.

The $\rho$-edge stability number $e s_{\rho}(G)$ of $G$ is the minimum number of edges of $G$ whose removal results in a graph $H \subseteq G$ with $\rho(H) \neq \rho(G)$ or with $E(H)=\emptyset$ (which implies $e s_{\rho}(G)=|E(G)|$ ).

The $\rho$-subdivision number $s d_{\rho}(G)$ of $G$ is the minimum number of edges of $G$ to be subdivided such that the resulting graph $H$ fulfills $\rho(H) \neq \rho(G)$. If such an edge set does not exist, then set $s d_{\rho}(G)=|E(G)|$.

Note that $e s_{\chi^{\prime \prime}}(G)=s d_{\chi^{\prime \prime}}(G)=0$ by definition if $G$ is empty, so we can assume in the following that $G$ is non-empty.

Subdividing an edge $e=u v$ of a graph $G$ creates a new graph $G_{e}$, in which a new vertex $w$ is added and the edge $e$ is removed and replaced by two new edges $u w$ and $w v$. We write $G_{E^{\prime}}$ for the graph obtained by subdividing all edges of $E^{\prime} \subseteq E(G)$. Note that each edge of $E^{\prime}$ is subdivided exactly once.

These two parameters based on edge removal or edge subdivision, respectively, were discussed in several papers, for example in [2,10] and more recently in [1,3,7-9] for the invariants chromatic number $\chi(G)$ and chromatic index $\chi^{\prime}(G)$.

In this paper we investigate the $\chi^{\prime \prime}$-edge stability number $e_{\chi^{\prime \prime}}(G)$, also called total chromatic edge stability number, and the $\chi^{\prime \prime}$-subdivision number $s d_{\chi^{\prime \prime}}(G)$, also called total chromatic subdivision number, with respect to the total chromatic number $\chi^{\prime \prime}(G)$ of $G$. Results on the total chromatic number are collected for example in [11] and first results on $s d_{\chi^{\prime \prime}}(G)$ can be found in [8].

A (proper) total coloring of $G$ is an assignment of colors to the vertices and edges of $G$ (together called the elements of $G$ ) such that neighbored elements - two adjacent vertices or two adjacent edges or a vertex and an incident edge-are colored differently. A $k$-total coloring is a proper total coloring with $k$ colors. The total chromatic number $\chi^{\prime \prime}(G)$ of $G$ is defined as the minimum $k$ in a $k$-total coloring of $G$. 
Obviously, $\chi^{\prime \prime}(G) \geq \Delta(G)+1$ by definition where $\Delta(G)$ is the maximum degree of $G$. The Total Coloring Conjecture states that $\chi^{\prime \prime}(G) \leq \Delta(G)+2$ for every graph $G$ (see [4], p. 282; [11], p. 4). Therefore, the truth of this conjecture would imply that $\chi^{\prime \prime}(G)$ attains one of two values for every graph $G$. Graphs $G$ are called type 1 graphs if $\chi^{\prime \prime}(G)=\Delta(G)+1$ and type 2 graphs if $\chi^{\prime \prime}(G)=\Delta(G)+2$, respectively. We define the invariant type $(G)=\chi^{\prime \prime}(G)-\Delta(G)$ which has a value in $\{1,2\}$ if the Total Coloring Conjecture is true.

We prove in this paper among others general upper and lower bounds for the total chromatic edge stability number $e s_{\chi^{\prime \prime}}(G)$ (general bounds for the total chromatic subdivision number $s d_{\chi^{\prime \prime}}(G)$ can be found in [8]). We also exactly determine $e s_{\chi^{\prime \prime}}(G)$ for specific classes of graphs such as acyclic graphs, cycles, complete graphs, and complete bipartite graphs. Moreover, we determine $s d_{\chi^{\prime \prime}}(G)$ for some graph classes, for example for even complete graphs and for complete bipartite graphs, extending results of [8].

\section{General results for the total chromatic edge stability number}

Some general results for the $\rho$-edge stability number are proved in [7]. If we apply the results on the total chromatic number, then we obtain the following statements. General proofs can be found in [7].

Proposition 2.1. Let $H$ be a spanning subgraph of G obtained from $G$ by removing kedges. Then es $\chi_{\chi^{\prime \prime}}(G) \leq e s_{\chi^{\prime \prime}}(H)+k$. Moreover, if $\chi^{\prime \prime}(H)<\chi^{\prime \prime}(G)$, then es $\chi^{\prime \prime}(G) \leq k$.

Corollary 2.1. If there is an edge set $E^{\prime} \subseteq E_{v}$ such that $\chi^{\prime \prime}\left(G-E_{v}\right)<\chi^{\prime \prime}\left(G-E^{\prime}\right)$ or $\chi^{\prime \prime}\left(G-E^{\prime}\right)<\chi^{\prime \prime}(G)$, where $E_{v}$ is the set of edges incident to $v$, then es $_{\chi^{\prime \prime}}(G) \leq d(v) \leq \Delta(G)$.

Theorem 12 of [7] states that the edge stability number for specific invariants can be computed by the edge stability numbers of its components. Applying this theorem to the total chromatic edge stability number gives the following result.

Proposition 2.2. Let $G=H_{1} \cup \cdots \cup H_{k}$ be the disjoint union of the subgraphs $H_{1}, \ldots, H_{k}$ whose indices and the integer $s$, $1 \leq s \leq k$, are defined such that $\chi^{\prime \prime}(G)=\chi^{\prime \prime}\left(H_{i}\right)$ if and only if $1 \leq i \leq s$. Then $e s_{\chi^{\prime \prime}}(G)=\sum_{i=1}^{s} e s_{\chi^{\prime \prime}}\left(H_{i}\right)$.

Therefore, we can assume in the following without loss of generality that $G$ is connected.

The following results from [7] give lower bounds based on not necessarily distinct subgraphs of a graph.

Theorem 2.1. Let $G$ be a non-empty graph with $\chi^{\prime \prime}(G)=k$. If $G$ contains s non-empty subgraphs $G_{1}, \ldots, G_{s}$ with $\chi^{\prime \prime}\left(G_{1}\right)=$ $\cdots=\chi^{\prime \prime}\left(G_{s}\right)=k$ such that $a \geq 0$ is the number of edges that occur in at least two of these subgraphs and $q \geq 1$ is the maximum number of these subgraphs with a common edge, then both $e s_{\chi^{\prime \prime}}(G) \geq \frac{1}{q} \sum_{i=1}^{s} e s_{\chi^{\prime \prime}}\left(G_{i}\right) \geq s / q$ and $e s_{\chi^{\prime \prime}}(G) \geq$ $\sum_{i=1}^{s} e s_{\chi^{\prime \prime}}\left(G_{i}\right)-a(q-1)$ hold.

Corollary 2.2. Let $G$ be a non-empty graph with $\chi^{\prime \prime}(G)=k$. If $G$ contains s non-empty subgraphs $G_{1}, \ldots, G_{s}$ with $\chi^{\prime \prime}\left(G_{1}\right)=$ $\cdots=\chi^{\prime \prime}\left(G_{s}\right)=k$ and pairwise disjoint edge sets, then $e_{\chi^{\prime \prime}}(G) \geq \sum_{i=1}^{s} e s_{\chi^{\prime \prime}}\left(G_{i}\right) \geq s$.

Corollary 2.3. If $H \subseteq G$ and $\chi^{\prime \prime}(H)=\chi^{\prime \prime}(G)$, then $e s_{\chi^{\prime \prime}}(H) \leq e s_{\chi^{\prime \prime}}(G)$.

Note that $e s_{\chi^{\prime \prime}}(H) \leq e s_{\chi^{\prime \prime}}(G)$ does not hold in general.

The $\rho$-edge stability number $e s_{\rho}(G)$ for $\rho(G)=\Delta(G)$ where $\Delta(G)$ is the maximum degree of $G$ was determined in [7]. It is proved that $e s_{\Delta}(G)=\left|V_{\Delta}\right|-\alpha^{\prime}\left(G\left[V_{\Delta}\right]\right)$ if $G$ is non-empty, where $V_{\Delta}$ is the set of vertices of $G$ of degree $\Delta(G), G\left[V_{\Delta}\right]$ is the subgraph of $G$ induced by $V_{\Delta}$, and $\alpha^{\prime}(G)$ is the edge independence number or matching number of $G$.

Lemma 2.1. If $G$ is a type 1 graph, then $e s_{\chi^{\prime \prime}}(G) \geq e s_{\Delta}(G)$.

Proof. If $G$ is non-empty, then there is a set $E^{\prime}$ of edges of $G$ such that $\left|E^{\prime}\right|=e s_{\chi^{\prime \prime}}(G)$ and

$$
\Delta\left(G-E^{\prime}\right)+1 \leq \chi^{\prime \prime}\left(G-E^{\prime}\right)<\chi^{\prime \prime}(G)=\Delta(G)+1 .
$$

It follows that $\Delta\left(G-E^{\prime}\right)<\Delta(G)$ which implies that $e s_{\chi^{\prime \prime}}(G)=\left|E^{\prime}\right| \geq e s_{\Delta}(G)$.

Let $\alpha^{\prime \prime}(G)$ be the total independence number of $G$, that is, the maximum number of pairwise not adjacent or incident elements in $G$. Let $t^{\prime \prime}(G)$ be the minimum number of elements in a color class of $G$ where the minimum is taken over all total colorings of $G$ with $\chi^{\prime \prime}(G)$ colors. Let $s$ be the minimum number of vertices of maximum degree in a color class with $t^{\prime \prime}(G)$ elements if $G$ is of type 1 and $s=0$ if $G$ is of type 2.

Proposition 2.3. $e s_{\chi^{\prime \prime}}(G) \leq t^{\prime \prime}(G)+s \leq\left\lfloor(|V(G)|+|E(G)|) / \chi^{\prime \prime}(G)\right\rfloor+s \leq \alpha^{\prime \prime}(G)+s$. 
Proof. Let $G$ be non-empty and consider a total coloring of $G$ with $\chi^{\prime \prime}(G)$ colors such that $C$ is a color class with $t^{\prime \prime}(G)$ elements and moreover $s$ vertices of maximum degree if $G$ is of type 1.

If $C$ does not contain any vertex, then removing all edges of $C$ reduces the total chromatic number, thus $e s_{\chi^{\prime \prime}}(G) \leq t^{\prime \prime}(G)$.

If $C$ contains vertices, then these will be recolored in an arbitrary order. Let $v \in C$. If there is a missing color in the closed neighborhood $N(v) \cup\{v\}$ of $v$, say color $\beta$, then removing the at most one edge of color $\beta$ incident to $v$ allows us to recolor vertex $v$ by color $\beta$. This is for example the case if $\chi^{\prime \prime}(G)>\Delta(G)+1$ or if $d(v)<\Delta(G)$. If otherwise there is no missing color in the closed neighborhood of $v$, then this implies that $\chi^{\prime \prime}(G)=\Delta(G)+1, d(v)=\Delta(G)$, and the neighbors of $v$ are colored pairwise differently. Select a color $\beta$ different from the color of $v$ and remove the edge of color $\beta$ incident to $v$ as well as the edge connecting $v$ with the neighbor of $\operatorname{color} \beta$. After removing these two edges, vertex $v$ can be recolored by color $\beta$. Repeat this recoloring for all other vertices of $C$ (which are independent). Removing the edges of $C$ again reduces the total chromatic number, which implies that $e s_{\chi^{\prime \prime}}(G) \leq t^{\prime \prime}(G)+s$.

By the pigeonhole principle, any total coloring of $G$ with $\chi^{\prime \prime}(G)$ colors has a color class with at most $\left\lfloor\frac{|V(G)|+|E(G)|}{\chi^{\prime \prime}(G)}\right\rfloor$ elements, which implies $t^{\prime \prime}(G) \leq\left\lfloor(|V(G)|+|E(G)|) / \chi^{\prime \prime}(G)\right\rfloor$. On the other hand, the lower bound

$$
\chi^{\prime \prime}(G) \geq(|V(G)|+|E(G)|) / \alpha^{\prime \prime}(G)
$$

implies the last inequality of the statement of the theorem.

In the next section it will be shown that for complete graphs $K_{n}$ of odd order, which are of type 1 , es $\chi^{\prime \prime}\left(K_{3}\right)=3>$ $\alpha^{\prime \prime}\left(K_{3}\right)=2$ holds, that is, the upper bound $\alpha^{\prime \prime}(G)+s$ is tight for $K_{3}$, and $e s_{\chi^{\prime \prime}}\left(K_{n}\right)=\alpha^{\prime \prime}\left(K_{n}\right)=(n+1) / 2$ if $n \geq 5$ is odd. The question arises for which type 1 graphs $G$ the upper bound for $e s_{\chi^{\prime \prime}}(G)$ can be improved to $\alpha^{\prime \prime}(G)$.

Theorem 2.2. If $G$ is a type 2 graph and the Total Coloring Conjecture is true, then es $\chi^{\prime \prime}(G)=\min \left\{e s_{\Delta}(G), e s_{\text {type }}(G)\right\}$.

Proof. Since $G$ is of type 2, the graph $G$ is not empty and the invariants $\Delta(G)$, type $(G)=2$, and $\chi^{\prime \prime}(G)=\Delta(G)+2$ can be reduced by edge removal.

By removing $e s_{\Delta}(G)$ edges $E^{\prime}$ such that $\Delta\left(G-E^{\prime}\right)<\Delta(G)$ we obtain

$$
\chi^{\prime \prime}\left(G-E^{\prime}\right) \leq \Delta\left(G-E^{\prime}\right)+2<\Delta(G)+2=\chi^{\prime \prime}(G)
$$

which implies $\left|E^{\prime}\right| \geq e s_{\chi^{\prime \prime}}(G)$. By removing $e s_{\text {type }}(G)$ edges $E^{\prime \prime}$ such that type $\left(G-E^{\prime \prime}\right)=1$ we obtain

$$
\chi^{\prime \prime}\left(G-E^{\prime \prime}\right)=\Delta\left(G-E^{\prime \prime}\right)+1 \leq \Delta(G)+1<\Delta(G)+2=\chi^{\prime \prime}(G)
$$

which implies $\left|E^{\prime \prime}\right| \geq e s_{\chi^{\prime \prime}}(G)$. It follows that $\min \left\{e s_{\Delta}(G), e s_{\text {type }}(G)\right\} \geq e s_{\chi^{\prime \prime}}(G)$.

Consider now a set $E^{\prime \prime \prime}$ of edges such that $\chi^{\prime \prime}\left(G-E^{\prime \prime \prime}\right)<\chi^{\prime \prime}(G)=\Delta(G)+2$. Then $G-E^{\prime \prime \prime}$ is of type 1 or $\Delta\left(G-E^{\prime \prime \prime}\right)<\Delta(G)$, since otherwise $\chi^{\prime \prime}\left(G-E^{\prime \prime \prime}\right)=\Delta\left(G-E^{\prime \prime \prime}\right)+2=\Delta(G)+2$, a contradiction. Therefore, $\left|E^{\prime \prime \prime}\right| \geq e s_{\text {type }}(G)$ or $\left|E^{\prime \prime \prime}\right| \geq e s_{\Delta}(G)$ which implies $e s_{\chi^{\prime \prime}}(G) \geq \min \left\{e s_{\Delta}(G), e s_{\text {type }}(G)\right\}$.

Yap ([11], p. 6) stated that if $\chi^{\prime \prime}(G)=t$ and if for any $t$-total coloring of $G$ every color class contains at least two edges of $G$, then $\chi^{\prime \prime}(G-e)=t$ for every edge $e$ of $G$, that is, $e s_{\chi^{\prime \prime}}(G) \geq 2$. This implies that a graph $G$ with $e s_{\chi^{\prime \prime}}(G)=1$ must have a $t$-total coloring with a color class with at most one edge.

\section{Total chromatic edge stability number for specific graph classes}

In this section we determine the total chromatic edge stability number of some well-known graph classes.

Proposition 3.1. For a path $P_{n}$ it holds that $e s_{\chi^{\prime \prime}}\left(P_{n}\right)=n-1$.

Proof. If $n \geq 2$ then $\chi^{\prime \prime}\left(P_{n}\right)=3$ and moreover $\chi^{\prime \prime}\left(P_{n}-E^{\prime}\right)=3$ for any set of edges $E^{\prime} \varsubsetneqq E\left(P_{n}\right)$, since $P_{n}-E^{\prime}$ contains a subgraph $P_{2}$ with total chromatic number 3. Therefore, es $\chi_{\chi^{\prime \prime}}\left(P_{n}\right)=\left|E\left(P_{n}\right)\right|=n-1$.

Proposition 3.2. For a cycle $C_{n}$ it holds that $e s_{\chi^{\prime \prime}}\left(C_{n}\right)=n$ if $3 \mid n$ and $e s_{\chi^{\prime \prime}}\left(C_{n}\right)=1$ if $3 \nmid n$.

Proof. It holds that $\chi^{\prime \prime}\left(C_{n}\right)=3$ if $3 \mid n$ and $\chi^{\prime \prime}\left(C_{n}\right)=4$ if $3 \nmid n$. If $3 \mid n$, then $\chi^{\prime \prime}\left(C_{n}-E^{\prime}\right)=\chi^{\prime \prime}\left(C_{n}\right)=3$ if $E^{\prime} \varsubsetneqq E\left(C_{n}\right)$ which implies that $e s_{\chi^{\prime \prime}}\left(C_{n}\right)=\left|E\left(C_{n}\right)\right|=n$. On the other hand, if $3 \nmid n$, then $C_{n}-e \cong P_{n}$ with $\chi^{\prime \prime}\left(C_{n}-e\right)=3<4=\chi^{\prime \prime}\left(C_{n}\right)$ for any edge $e$ of the cycle, which implies that $e s_{\chi^{\prime \prime}}\left(C_{n}\right)=1$.

Proposition 3.3. For an acyclic graph $G$ it holds that es $\chi_{\chi^{\prime \prime}}(G)=|E(G)|$ if $\Delta(G) \leq 2$ and es $\chi_{\chi^{\prime \prime}}(G)=e s_{\Delta}(G)$ if $\Delta(G) \geq 3$. 
Proof. For an acyclic graph $G$ it holds that $\chi^{\prime \prime}(G)=\Delta(G)+2=3$ if $\Delta(G)=1$ and $\chi^{\prime \prime}(G)=\Delta(G)+1$ if $\Delta(G) \neq 1$ (proof by induction).

By Propositions 2.2 and 3.1, es $\chi_{\chi^{\prime \prime}}(G)=|E(G)|$ if $\Delta(G) \leq 2$, so assume in the following that $\Delta(G) \geq 3$. Since $G$ is a type 1 graph, $e s_{\chi^{\prime \prime}}(G) \geq e s_{\Delta}(G)$ by Lemma 2.1. On the other hand, let $E^{\prime}$ be a set of edges of $G$ such that $\left|E^{\prime}\right|=e s_{\Delta}(G)$ and $\Delta\left(G-E^{\prime}\right)<\Delta(G)$. By the minimality of $E^{\prime}, \Delta\left(G-E^{\prime}\right)=\Delta(G)-1 \geq 2$ which implies that $\chi^{\prime \prime}\left(G-E^{\prime}\right)=\Delta\left(G-E^{\prime}\right)+1<$ $\Delta(G)+1=\chi^{\prime \prime}(G)$ since $G-E^{\prime}$ is acyclic. Therefore, $e s_{\chi^{\prime \prime}}(G) \leq\left|E^{\prime}\right|=e s_{\Delta}(G)$ by the minimality of the total chromatic edge stability number.

This result implies that the lower bound of Lemma 2.1 is tight if $G$ is an acyclic graph with $\Delta(G) \geq 3$. On the other hand, the difference between $e s_{\chi^{\prime \prime}}(G)$ and $e s_{\Delta}(G)$ may be arbitrarily large, e.g., for cycles of orders divisible by 3 or for unions of paths of order at least 3. For example, if $G \cong k P_{3}$, then $e s_{\chi^{\prime \prime}}(G)=|E(G)|=2 k$ and $e s_{\Delta}(G)=k$.

We now consider complete graphs $K_{n}$.

Proposition 3.4. $e s_{\chi^{\prime \prime}}\left(K_{n}\right)= \begin{cases}3 & \text { if } n=3, \\ (n+1) / 2 & \text { if } n \geq 5 \text { is odd, } \\ \lfloor(n+2) / 4\rfloor & \text { if } n \text { is even. }\end{cases}$

Proof. 1. If $n=3$, then $e s_{\chi^{\prime \prime}}\left(K_{3}\right)=e s_{\chi^{\prime \prime}}\left(C_{3}\right)=3$ by Proposition 3.2.

2. If $n \geq 5$ is odd, then $K_{n}$ is of type 1 , thus $e s_{\chi^{\prime \prime}}\left(K_{n}\right) \geq e s_{\Delta}\left(K_{n}\right)=(n+1) / 2$ by Lemma 2.1 .

Theorem 6.24 of [11] states that if $G$ is a graph of odd order $n=2 k+1$ with maximum degree $\Delta(G)=n-2$ and if $w$ is a vertex of minimum degree in $G$, then $G$ is of type 1 if and only if $\left.|E(\overline{G-w})|+\alpha^{\prime}(\overline{G-w})\right) \geq k$ where $\bar{G}=\left(V,\left(\begin{array}{c}V \\ 2\end{array}\right) \backslash E\right)$ is the complement of $G=(V, E)$.

Denote the vertices of $K_{n}$ by $v_{1}, \ldots, v_{n}$ and let $G=K_{n}-E^{\prime}$ where $E^{\prime}$ contains the $k=(n-1) / 2$ independent edges $v_{1} v_{n-1}, v_{2} v_{n-2}, \ldots, v_{k} v_{k+1}$ and additionally edge $v_{n-1} v_{n}$. It follows that $w=v_{n-1}$ is the only vertex of minimum degree $n-3$ in $G$ and therefore $\left.|E(\overline{G-w})|=\alpha^{\prime}(\overline{G-w})\right)=\left|E^{\prime}\right|-2=k-1$. By the above result, $G$ is of type 1 if and only if $2(k-1) \geq k$, that is, $k \geq 2$ which is equivalent to $n \geq 5$.

If $n \geq 5$ is odd, then it follows that $\chi^{\prime \prime}(G)=\Delta(G)+1=n-1<n=\chi^{\prime \prime}\left(K_{n}\right)$, that is, $e s_{\chi^{\prime \prime}}\left(K_{n}\right) \leq\left|E^{\prime}\right|=(n+1) / 2$.

3. Hilton [5] proved the following result on the total chromatic number of complete graphs of even order from which edges are removed:

Let $n \geq 2$ even, $E^{\prime} \subseteq E\left(K_{n}\right), j$ be the maximum number of independent edges in $E^{\prime}$. Then $\chi^{\prime \prime}\left(K_{n}-E^{\prime}\right)=n+1$ if and only if $\left|E^{\prime}\right|+j \leq n / 2-1$.

This means that an edge set $E^{\prime} \subseteq E\left(K_{n}\right)$ with $\left|E^{\prime}\right|=e x_{\chi^{\prime \prime}}\left(K_{n}\right)$ and $\chi^{\prime \prime}\left(K_{n}-E^{\prime}\right)<\chi^{\prime \prime}\left(K_{n}\right)=n+1$ must fulfill $\left|E^{\prime}\right|+j>n / 2-1$. Note that the cardinality of $E^{\prime}$ is minimal if all edges are independent, that is, $j=\left|E^{\prime}\right|$ (for $\left|E^{\prime}\right| \leq$ $\alpha^{\prime}\left(K_{n}\right)=n / 2$ edges). Therefore, we obtain as necessary condition that $\left|E^{\prime}\right|>(n-2) / 4$, that is, $\left|E^{\prime}\right| \geq\lfloor(n+2) / 4\rfloor$.

Indeed, removing $n / 4$ independent edges if $n \equiv 0(\bmod 4)$ and $(n+2) / 4$ independent edges if $n \equiv 2(\bmod 4)$, that is, $\lfloor(n+2) / 4\rfloor$ independent edges, gives a graph $K_{n}-E^{\prime}$ for which $\left|E^{\prime}\right|+j>n / 2-1$ holds, which implies $\chi^{\prime \prime}\left(K_{n}-E^{\prime}\right)<$ $n+1=\chi^{\prime \prime}\left(K_{n}\right)$ by the theorem of Hilton.

It follows that $e s_{\chi^{\prime \prime}}\left(K_{n}\right)=\lfloor(n+2) / 4\rfloor$ if $n$ is even.

The last result of this proposition shows that also for type 2 graphs $G$ the difference between $e s_{\chi^{\prime \prime}}(G)$ and $e s_{\Delta}(G)$ may be arbitrarily large, since $e s_{\Delta}\left(K_{n}\right)=\lceil n / 2\rceil$ for $n \geq 2$.

A wheel $W_{n}, n \geq 3$, is the join of a cycle $C_{n}$ and a singleton $K_{1}$.

Proposition 3.5. For a wheel $W_{n}, n \geq 3$, it holds that es $\chi^{\prime \prime}\left(W_{n}\right)=1$.

Proof. If $n=3$, then $W_{3} \cong K_{4}$, thus $e s_{\chi^{\prime \prime}}\left(W_{3}\right)=1$ by Proposition 3.4.

If $n \geq 4$, then $W_{n}$ is of type 1: Denote the vertices of $W_{n}$ by $v_{1}, \ldots, v_{n}$ for the vertices of the cycle and $z$ for the central vertex. Color vertex $v_{i}$ by color $i$ and $z$ by color $n+1$, edge $v_{i} z$ by color $i-1$ and $v_{i} v_{i+1}$ by color $i-2, i=1, \ldots, n$, where the indices and colors are considered modulo $n$ in the set $\{1, \ldots, n\}$. Note that color $n+1$ is only used to color vertex $z$.

The graph $W_{n}-v_{1} z$ is also of type 1: At first color the elements of $W_{n}-v_{1} z$ as above, then recolor edge $v_{1} v_{n}$ and vertex $z$ by color $n$ and vertex $v_{n}$ by color $n-2$, in order to obtain a proper $n$-total coloring of $W_{n}-v_{1} z$. It follows that $e s_{\chi^{\prime \prime}}\left(W_{n}\right)=1$ if $n \geq 4$.

For complete bipartite graphs $K_{a, b}$ it holds that $K_{a, b}$ is of type 1 if $a \neq b$ and of type 2 if $a=b$. In order to determine the total chromatic edge stability number of $K_{a, b}$ we use the following lemma. 
Lemma 3.1. Let $G$ be a bipartite graph. If all vertices of maximum degree are in one partition set, then $G$ is of type 1.

Proof. Color the edges of the bipartite graph with colors $1, \ldots, \Delta(G)$ which is possible by the Theorem of König (see [4], p. 257). Color the vertices of the partition set containing all vertices of maximum degree by color $\Delta(G)+1$. Every vertex $v$ of the second partition set has a degree smaller than $\Delta(G)$, thus there is a color from $\{1, \ldots, \Delta(G)\}$ missing at its incident edges. This color can be used to color $v$ since the vertices of a partition set are pairwise non-adjacent.

Note that this condition is not necessary, since, for example, $C_{6}$ is a regular bipartite graph of type 1.

Proposition 3.6. For a complete bipartite graph $K_{a, b}$, $a \leq b$, it holds that $e s_{\chi^{\prime \prime}}\left(K_{1,2}\right)=2$, es $\chi_{\chi^{\prime \prime}}\left(K_{a, b}\right)=a$ if $a<b$, $(a, b) \neq(1,2)$, and $e_{\chi^{\prime \prime}}\left(K_{a, a}\right)=\lceil a / 2\rceil$.

Proof. 1. By Proposition 3.1, es $\chi_{\chi^{\prime \prime}}\left(K_{1,2}\right)=e s_{\chi^{\prime \prime}}\left(P_{3}\right)=2$.

2. If $a<b$, then $K_{a, b}$ is of type 1 by Lemma 3.1, thus $e s_{\chi^{\prime \prime}}\left(K_{a, b}\right) \geq e s_{\Delta}\left(K_{a, b}\right)=a$ by Lemma 2.1 .

If $a<b-1$, then removing a set $E^{\prime}$ of $a$ independent edges gives a bipartite graph of maximum degree $b-1>a$ which is of type 1 by Lemma 3.1. Therefore, $\chi^{\prime \prime}\left(K_{a, b}-E^{\prime}\right)=\Delta\left(K_{a, b}-E^{\prime}\right)+1=b<b+1=\chi^{\prime \prime}\left(K_{a, b}\right)$ which implies that $e s_{\chi^{\prime \prime}}\left(K_{a, b}\right) \leq a$, that is, $e s_{\chi^{\prime \prime}}\left(K_{a, b}\right)=a$ if $a<b-1$.

3. If $b=a+1$ and $a \geq 2$, then denote the partition sets of $K_{a, a+1}$ by $V_{1}=\left\{v_{1}, \ldots, v_{a}\right\}$ and $V_{2}=\left\{u_{1}, \ldots, u_{a+1}\right\}$. Consider the edge coloring $c$ of $K_{a, a+1}$ given by $c\left(v_{i} u_{j}\right)=1+(i+j-2) \bmod (a+1), i \in\{1, \ldots, a\}, j \in\{1, \ldots, a+1\}$. Note that vertex $u_{1}$ is incident to edges of color $1, \ldots, a$ and thus can be colored by color $a+1$.

If $a$ is even then the edge $v_{i} u_{i+1}$ is colored by $2 i$ for $i \in\{1, \ldots, a / 2\}$ and by $2 i-a-1$ for $i \in\{a / 2+1, \ldots, a\}$, that is, the $a$ edges $E^{\prime}=\left\{v_{i} u_{i+1}: i \in\{1, \ldots, a\}\right\}$ are colored by pairwise different colors $\{1, \ldots, a\}$.

Removing $E^{\prime}$ and coloring the vertices $v_{i}$ and $u_{i+1}$ by the edge color $c\left(v_{i} u_{i+1}\right), i=1, \ldots, a$, completes an $(a+1)$-total coloring of $K_{a, a+1}-E^{\prime}$, which implies that $e s_{\chi^{\prime \prime}}\left(K_{a, a+1}\right) \leq\left|E^{\prime}\right|=a$, that is, $e s_{\chi^{\prime \prime}}\left(K_{a, a+1}\right)=a$.

If $a \geq 3$ is odd then we need to interchange two edge colors in $c$. Note that the edges incident with $u_{2}$ are colored by $2, \ldots, a+1$, that is, color 1 is missing at $u_{2}$, and that the edge $v_{(a+1) / 2} u_{2}$ is colored by color $(a+3) / 2$. At vertex $u_{(a+5) / 2}$, color $(a+3) / 2$ is missing and the edge $v_{(a+1) / 2} u_{(a+5) / 2}$ is colored by color 1 , that is, we can interchange the colors of these two edges incident to vertex $v_{(a+1) / 2}$. After this recoloring, edge $v_{(a+1) / 2} u_{2}$ is colored by 1 , edges $v_{i} u_{i+2}$ are colored by color $2 i+1$ for $i \in\{1, \ldots,(a-1) / 2\}$, and edges $v_{i} u_{i+1}$ are colored by $2 i-a-1$ for $i \in\{(a+3) / 2, \ldots, a\}$, that is, these $a$ edges are again colored by pairwise different colors $\{1, \ldots, a\}$, and the proof can be analogously completed as in the case of even $a$.

4. Consider now $K_{a, a}$. Since $e s_{\chi^{\prime \prime}}\left(K_{1,1}\right)=e s_{\chi^{\prime \prime}}\left(K_{2}\right)=1$ and $e s_{\chi^{\prime \prime}}\left(K_{2,2}\right)=e s_{\chi^{\prime \prime}}\left(C_{4}\right)=1$, the assumption holds for $a \in\{1,2\}$. Let in the following $a \geq 3$.

Let $E^{\prime}$ be a set of edges such that $\left|E^{\prime}\right|=e s_{\chi^{\prime \prime}}\left(K_{a, a}\right)$ and $\chi^{\prime \prime}\left(K_{a, a}-E^{\prime}\right)<\chi^{\prime \prime}\left(K_{a, a}\right)=a+2$. Denote by $V_{1}, V_{2}$ the partition sets of $K_{a, a}$ and let $X_{1} \subseteq V_{1}$ and $X_{2} \subseteq V_{2}$ be the sets of vertices incident to the edges of $E^{\prime}$. Without loss of generality, assume $\left|X_{1}\right| \leq\left|X_{2}\right|$.

Consider a total coloring of $K_{a, a}-E^{\prime}$ with $a+1$ colors. Each color class contains at most $a$ elements (say for each $v \in V_{i}$ either $v$ or an edge incident to $v$ ) and possibly some additional vertices of $X_{3-i}$ if the color class does not contain vertices of $V_{i} \backslash X_{i}, i \in\{1,2\}$. Taking all color classes into account, there may be at most $\left|X_{1}\right|$ vertices added in such a way, therefore the number of elements in all $a+1$ color classes is at most $(a+1) a+\left|X_{1}\right|$. On the other hand, $K_{a, a}-E^{\prime}$ has $2 a$ vertices and $a^{2}-\left|E^{\prime}\right|$ edges, so it must hold that $(a+1) a+\left|X_{1}\right| \geq 2 a+a^{2}-\left|E^{\prime}\right|$ which is equivalent to $\left|X_{1}\right|+\left|E^{\prime}\right| \geq a$. Since $\left|X_{1}\right| \leq\left|E^{\prime}\right|$, we obtain the lower bound es $_{\chi^{\prime \prime}}\left(K_{a, a}\right)=\left|E^{\prime}\right| \geq a / 2$.

We will now prove that $e s_{\chi^{\prime \prime}}\left(K_{a, a}\right) \leq\lceil a / 2\rceil$. Denote the vertices of $K_{a, a}$ by $V_{1}=\left\{v_{1}, \ldots, v_{a}\right\}$ and $V_{2}=\left\{u_{1}, \ldots, u_{a}\right\}$ and set $X_{1}=\left\{v_{1}, \ldots, v_{l}\right\}$ and $X_{2}=\left\{u_{1}, \ldots, u_{l}\right\}$ where $l=\lceil a / 2\rceil$.

We consider the following edge coloring of $K_{a, a}$ :

$$
c\left(v_{i} u_{j}\right)=1+(l+j-i) \bmod a, \quad i, j \in\{1, \ldots, a\} .
$$

The edges $\left\{v_{l+j} u_{j}: j=1, \ldots, l\right\}$ if $a$ is even or $\left\{v_{l+j} u_{j}: j=1, \ldots, l-1\right\}$ if $a$ is odd, respectively, are colored by color 1. We recolor these edges by color $a+1$ and color the vertices of $V_{1} \backslash X_{1}=\left\{v_{l+1}, \ldots, v_{a}\right\}$ by 1 and the vertices of $V_{2} \backslash X_{2}=\left\{u_{l+1}, \ldots, u_{a}\right\}$ by $a+1$. 
In the following we determine a set $E^{\prime}$ of $l$ independent and pairwise distinctly colored edges from the subgraph $K_{l, l}$ of $K_{a, a}$ induced by $X_{1} \cup X_{2}$.

If $l \geq 3$ is odd, then the edges of $E^{\prime}=\left\{v_{i} u_{2 i-1}: i=1, \ldots, l\right\}$ (indices considered modulo $l$ ) are independent and colored by the colors $l+1, \ldots, l+(l+1) / 2$ and $(l+3) / 2, \ldots, l$ which are pairwise different and different from $1, a+1$.

If $l \geq 2$ is even, then the edges of $E^{\prime}=\left\{v_{i} u_{2 i}: i=1, \ldots, l / 2\right\} \cup\left\{v_{i} u_{2 i-l-1}: i=l / 2+1, \ldots, l\right\}$ are independent and colored by the colors $l+2, \ldots, l+l / 2+1$ and $l / 2+1, \ldots, l$, respectively, which are pairwise different and different from $1, a+1$ if $a \neq 3$.

After removing the $l$ selected edges of $E^{\prime}$, their respective end-vertices can be colored by the color of the removed edge. If $a$ is odd, then vertex $u_{l}$ will be recolored by color $a+1$. This completes a total coloring of $K_{a, a}-E^{\prime}$ with only $a+1$ colors. It follows that $e_{\chi^{\prime \prime}}\left(K_{a, a}\right) \leq\left|E^{\prime}\right|=\lceil a / 2\rceil$.

Observe that the above proof is constructive. The described total coloring of $K_{a, a}-E^{\prime}$ will be used in Proposition 4.6. Let us note that it is also possible to prove the case $e s_{\chi^{\prime \prime}}\left(K_{a, a}\right)=\lceil a / 2\rceil$ using a theorem of Hilton [6] on complete bipartite graphs. This result states that if $E^{\prime} \subseteq E\left(K_{a, a}\right)$ and $j$ is the maximum number of independent edges in $E^{\prime}$, then $\chi^{\prime \prime}\left(K_{a, a}-\right.$ $\left.E^{\prime}\right)=a+2$ if and only if $\left|E^{\prime}\right|+j \leq a-1$.

A generalized $\theta$-graph $\theta_{l_{1}, \ldots, l_{m}}, l_{1} \leq \cdots \leq l_{m}$, is a graph with two vertices connected by $m$ internally disjoint paths of length $l_{1}, \ldots, l_{m}$. If $m=1$, then $\theta_{l_{1}}$ is a path of length $l_{1}$ and if $m=2$, then $\theta_{l_{1}, l_{2}}$ is a cycle of length $l_{1}+l_{2}$ which have been discussed in the above propositions, so in the following we may assume $m \geq 3$. Note that the two vertices of maximum degree $m$ are adjacent if and only if $l_{1}=1$.

Proposition 3.7. If $G$ is a generalized $\theta$-graph $\theta_{l_{1}, \ldots, l_{m}}$ with $m \geq 3$, then es $_{\chi^{\prime \prime}}(G)=1$ if $l_{1}=1$ and either $m \geq 4$ or $m=3$, $3 \mid l_{2}+l_{3}$. In all other cases, es $\chi^{\prime \prime}(G)=2$.

Proof. In [8] it was shown that all generalized $\theta$-graphs with $m \geq 3$ paths are of type 1, thus $e s_{\chi^{\prime \prime}}(G) \geq e s_{\Delta}(G)$ by Lemma 2.1, where $e s_{\Delta}(G)=1$ if $l_{1}=1$ and $e s_{\Delta}(G)=2$ if $l_{1}>1$.

1. $l_{1}=1$ : Denote by $e$ the edge connecting the two vertices of maximum degree. If $m \geq 4$, then removing $e$ gives a generalized $\theta$-graph with $m-1 \geq 3$ paths which is of type 1 . If $m=3$ and $l_{2}+l_{3}$ is divisible by 3 , then removing $e$ gives a cycle $C_{l_{2}+l_{3}}$ which is also of type 1. Therefore, $\chi^{\prime \prime}(G-e)=\Delta(G-e)+1=m<m+1=\chi^{\prime \prime}(G)$, that is, $e s_{\chi^{\prime \prime}}(G)=1$ in these two cases.

If $m=3$ and $l_{2}+l_{3}$ is not divisible by 3 , then removing a single edge gives a cycle $C_{l_{2}+l_{3}}$ of type 2 or a graph of maximum degree 3 , that is, the total chromatic number does not decrease, and $e s_{\chi^{\prime \prime}}(G) \geq 2$ follows. On the other hand, removing $e$ and a second arbitrary edge gives a path $P_{l_{2}+l_{3}}$ which is of type 1 , thus $e s_{\chi^{\prime \prime}}(G)=2$ holds in this case.

2. $l_{1}>1$ : If $m \geq 4$ then removing the first and the last edge of one of the $m$ paths of $G$ gives the union $H$ of a path and of a generalized $\theta$-graph with $m-1 \geq 3$ paths which is of type 1 . If $m=3$ then removing two independent edges incident with a vertex of maximum degree each gives a path $H$ which is of type 1 . In either case, $\chi^{\prime \prime}(H)=\Delta(H)+1=$ $m<m+1=\chi^{\prime \prime}(G)$ which implies that $e s_{\chi^{\prime \prime}}(G) \leq 2$ and therefore $e s_{\chi^{\prime \prime}}(G)=2$.

\section{Total chromatic subdivision number}

The total chromatic subdivison number $s d_{\chi^{\prime \prime}}(G)$ of a graph $G$ is the minimum number of edges of $G$ whose subdivision results in a graph $H$ with $\chi^{\prime \prime}(H) \neq \chi^{\prime \prime}(G)$. If such an edge set does not exist, then $s d_{\chi^{\prime \prime}}(G)=|E(G)|$ (see Introduction).

This invariant was first studied in [8]. In that paper $s d_{\chi^{\prime \prime}}(G)$ was determined for several classes of graphs but the determination for type 2 graphs remained nearly completely open. For example, it was proved that $s d_{\chi^{\prime \prime}}(G)=|E(G)|$ if $G$ is acyclic. For cycles $C_{n}$ it holds that $s d_{\chi^{\prime \prime}}\left(C_{n}\right)=1$ if $n \equiv 0(\bmod 3)$ or $n \equiv 2(\bmod 3)$, and $s d_{\chi^{\prime \prime}}\left(C_{n}\right)=2$ if $n \equiv 1(\bmod 3)$. If $G$ is a generalized $\theta$-graph consisting of $m \geq 3$ paths, then $s d_{\chi^{\prime \prime}}(G)=|E(G)|$. For complete graphs $K_{n}$ it holds that $s d_{\chi^{\prime \prime}}\left(K_{1}\right)=0$ and $s d_{\chi^{\prime \prime}}\left(K_{n}\right)=1$ if $n=2$ or $n \geq 3$ is odd. Note that $K_{n}$ is of type 1 if $n$ is odd.

We will apply the following proposition for studying $s d_{\chi^{\prime \prime}}(G)$ for type 2 graphs. The result says that if the Total Coloring Conjecture holds for a graph $G$, then it also holds for the resulting graph after subdividing an arbitrary number of edges of $G$. We give a direct proof of this known result.

Proposition 4.1. If $\chi^{\prime \prime}(G) \leq \Delta(G)+2$ then $\chi^{\prime \prime}\left(G_{E^{\prime}}\right) \leq \Delta\left(G_{E^{\prime}}\right)+2$ for any set $E^{\prime}$ of edges of $G$. 
Proof. If $\Delta(G) \leq 2$ then $G$ consists of components which are paths or cycles. Subdividing edges of $G$ gives longer paths or cycles. Since the Total Coloring Conjecture holds for paths and for cylces, it holds for $G$ and for $G_{E^{\prime}}$.

Therefore, we may assume in the following that $\Delta(G) \geq 3$, which implies that $\Delta\left(G_{E^{\prime}}\right)=\Delta(G)$.

Let $e=u v$ be an arbitrary edge of $G$ and $w$ be the subdivision vertex in $G_{e}$. Consider a $(\Delta(G)+2)$-total coloring of $G$. Color all elements of $G_{e}$ except $w, u w, v w$ as in $G$, then color $u w$ and $v w$ by different colors missing at $u$ and at $w$, respectively, which is possible since at most $\Delta(G)$ colors were used at $u, w$. Finally, color $w$ by a color different from the colors of the 4 incident or adjacent elements which is possible since there are $\Delta(G)+2 \geq 5$ available colors. Therefore, $\chi^{\prime \prime}\left(G_{e}\right) \leq \Delta(G)+2$.

Applying this result on the graphs obtained by successively subdividing a single edge of $E^{\prime}$, say $G, G_{e_{1}}, G_{\left\{e_{1}, e_{2}\right\}}, \ldots, G_{E^{\prime}}$, we obtain that $\chi^{\prime \prime}\left(G_{E^{\prime}}\right) \leq \Delta(G)+2=\Delta\left(G_{E^{\prime}}\right)+2$.

Proposition 4.2. If $G$ is of type 2, then $e \chi_{\chi^{\prime \prime}}(G) \leq s d_{\chi^{\prime \prime}}(G)$.

Proof. If $\chi^{\prime \prime}(G)$ does not change by edge subdivisions, then $e s_{\chi^{\prime \prime}}(G) \leq|E(G)|=s d_{\chi^{\prime \prime}}(G)$. This holds for $\Delta(G)=1$, therefore we may assume in the following that $\Delta(G) \geq 2$ which implies that $\Delta\left(G_{E^{\prime}}\right)=\Delta(G)$ for any set $E^{\prime}$ of edges.

Let $E^{\prime} \subseteq E(G)$ be a set of edges such that $\left|E^{\prime}\right|=s d_{\chi^{\prime \prime}}(G)$ and $\chi^{\prime \prime}\left(G_{E^{\prime}}\right) \neq \chi^{\prime \prime}(G)$. By Proposition $4.1, \Delta(G)+1 \leq \chi^{\prime \prime}\left(G_{E^{\prime}}\right) \leq$ $\Delta(G)+2=\chi^{\prime \prime}(G)$ which implies that $\chi^{\prime \prime}\left(G_{E^{\prime}}\right)=\Delta(G)+1<\chi^{\prime \prime}(G)$.

Since $G-E^{\prime}$ is a subgraph of $G_{E^{\prime}}$, it holds that $\chi^{\prime \prime}\left(G-E^{\prime}\right) \leq \chi^{\prime \prime}\left(G_{E^{\prime}}\right)<\chi^{\prime \prime}(G)$ which implies that $e s_{\chi^{\prime \prime}}(G) \leq\left|E^{\prime}\right|=$ $s d_{\chi^{\prime \prime}}(G)$.

We now consider complete graphs of even order which are of type $2: \chi^{\prime \prime}\left(K_{2 k}\right)=\Delta\left(K_{2 k}\right)+2=2 k+1$.

Proposition 4.3. For complete graphs of even order it holds that $s d_{\chi^{\prime \prime}}\left(K_{2 k}\right)=\lfloor(k+1) / 2\rfloor, k \in \mathbb{N}$.

Proof. 1. By definition, $s d_{\chi^{\prime \prime}}\left(K_{2}\right)=1$. Consider as an example the complete graph $K=K_{4}$, say with vertices $v_{1}, v_{2}, v_{3}, v_{4}$, and the graph $K_{E^{\prime}}$ obtained by subdividing the edge of $E^{\prime}=\left\{v_{1} v_{2}\right\}$ with subdivision vertex $w_{1}$. Then coloring $v_{1} w_{1}, v_{2} v_{4}, v_{3}$ by color $1, v_{1}, v_{2}, v_{3} v_{4}$ by color $2, v_{1} v_{3}, w_{1} v_{2}, v_{4}$ by color 3 , and $v_{1} v_{4}, v_{2} v_{3}, w_{1}$ by color 4 gives a total coloring of $K_{E^{\prime}}$ with $\Delta\left(K_{E^{\prime}}\right)+1=4$ colors. It follows that $s d_{\chi^{\prime \prime}}\left(K_{4}\right)=1$. This coloring will be generalized in the proof below.

2. The lower bound $\lfloor(k+1) / 2\rfloor=e s_{\chi^{\prime \prime}}\left(K_{2 k}\right) \leq s d_{\chi^{\prime \prime}}\left(K_{2 k}\right)$ for $k \in \mathbb{N}$ follows directly from Propositions 3.4 and 4.2 since $K_{2 k}$ is of type 2 .

3. We will now prove the upper bound $s d_{\chi^{\prime \prime}}\left(K_{2 k}\right) \leq\lfloor(k+1) / 2\rfloor$ for $k \geq 2$.

Let $K=K_{2 k}$ and $V(K)=\left\{v_{1}, \ldots, v_{2 k}\right\}$. Consider the edge coloring $c$ of $K$ with $2 k$ colors defined by $c\left(v_{i} v_{j}\right)=((i+j-$ $2) \bmod 2 k)+1,1 \leq i<j \leq 2 k$. Since $K$ is $(2 k-1)$-regular, a color is missing at each vertex, namely color $2 i-1$ at vertex $v_{i}$ and $v_{k+i}, 1 \leq i \leq k$. These are the odd colors $1,3, \ldots, 2 k-1$, each occurring twice.

Our aim is to select a set $E^{\prime}$ of $\lfloor(k+1) / 2\rfloor$ independent edges which are colored pairwise differently with even colors. With the given edge coloring we can select the edges $E^{\prime}=\left\{v_{2 t-1} v_{2 t}: 1 \leq t \leq\lfloor(k+1) / 2\rfloor\right\}$ which are obviously independent. Their colors are $C^{\prime}=\left\{c\left(v_{2 t-1} v_{2 t}\right): 1 \leq t \leq\lfloor(k+1) / 2\rfloor\right\}=\{4 t-2: 1 \leq t \leq\lfloor(k+1) / 2\rfloor\}$ which are all colors congruent to 2 modulo 4 from 2 to $2 k-2$ if $k$ is even or from 2 to $2 k$ if $k$ is odd, respectively. Note also that each edge from $E^{\prime}$ connects consecutive vertices, thus their respective missing colors are consecutive odd numbers or $2 k-1$ and 1 for the last edge $v_{k} v_{k+1}$ if $k$ is odd. In any case, the two missing colors are different.

We now consider the graph $K_{E^{\prime}}$ in which edge $v_{2 t-1} v_{2 t}$ of $E^{\prime}$ is subdivided by vertex $w_{t}, 1 \leq t \leq\lfloor(k+1) / 2\rfloor$. We extend the edge coloring $c$ to a total coloring $c^{\prime}$ of $K_{E^{\prime}}$ as follows: Color each non-subdivided edge as in $c$. Color $v_{2 t-1} w_{t}$ and $w_{t} v_{2 t}$ with the missing color of $v_{2 t-1}$ and of $v_{2 t}$, respectively, which are different, for $1 \leq t \leq\lfloor(k+1) / 2\rfloor$. Color the vertices $v_{2 t-1}$ and $v_{2 t}$ with the even color $c\left(v_{2 t-1} v_{2 t}\right)=4 t-2$ and color $w_{t}$ with a possible of the $2 k$ colors which exists for $k \geq 2$ since the neighbored elements of $w_{t}$ only use 3 colors, $1 \leq t \leq\lfloor(k+1) / 2\rfloor$. Color the remaining vertices $v_{k+1+(k \bmod 2)}, \ldots, v_{2 k}$ with the corresponding missing colors which are odd and pairwise different by construction.

Since $c^{\prime}$ is a proper total coloring of $K_{E^{\prime}}$ with $2 k$ colors, $\chi^{\prime \prime}\left(K_{E^{\prime}}\right)=\Delta\left(K_{E^{\prime}}\right)+1=2 k$ and $s d_{\chi^{\prime \prime}}\left(K_{2 k}\right) \leq\left|E^{\prime}\right|=\lfloor(k+1) / 2\rfloor$ follow.

The following result was proved in [8].

Proposition 4.4 ([8]). If $G$ is a type 1 graph with no adjacent vertices of maximum degree, then $s d_{\chi^{\prime \prime}}(G)=|E(G)|$, with the possible exception that $\Delta(G)=3$ and $G$ has vertices of degree 2 which are adjacent to two vertices of maximum degree.

Proposition 4.5. For a wheel $W_{n}$ it holds that $s d_{\chi^{\prime \prime}}\left(W_{3}\right)=1$ and $s d_{\chi^{\prime \prime}}\left(W_{n}\right)=2 n$ if $n \geq 4$. 
Proof. If $n=3$, then $W_{3} \cong K_{4}$, thus $s d_{\chi^{\prime \prime}}\left(W_{3}\right)=1$ by Proposition 4.3 .

If $n \geq 4$, then $W_{n}$ is of type 1 (see proof of Proposition 3.5) with only one vertex of maximum degree. Therefore, $s d_{\chi^{\prime \prime}}\left(W_{n}\right)=\left|E\left(W_{n}\right)\right|=2 n$ by Proposition 4.4.

Consider now complete bipartite graphs.

Proposition 4.6. For a complete bipartite graph $K_{a, b}, a \leq b$, it holds that $s d_{\chi^{\prime \prime}}\left(K_{a, b}\right)=\left|E\left(K_{a, b}\right)\right|=a b$ if $a<b$, sd $d_{\chi^{\prime \prime}}\left(K_{2,2}\right)=$ 2 , and $s d_{\chi^{\prime \prime}}\left(K_{a, a}\right)=\lceil a / 2\rceil$ if $a \neq 2$.

Proof. If $a<b$, then $K_{a, b}$ is of type 1 with no adjacent vertices of maximum degree. Thus Proposition 4.4 implies that $s d_{\chi^{\prime \prime}}\left(K_{a, b}\right)=\left|E\left(K_{a, b}\right)\right|$ with the possible exception of $K_{2,3}$. Since $K_{2,3} \cong \theta_{2,2,2}$, it also holds for this graph that $s d_{\chi^{\prime \prime}}\left(K_{2,3}\right)=$ $\left|E\left(K_{2,3}\right)\right|$ by Proposition 22 of [8] where the total chromatic subdivision number of generalized $\theta$-graphs is determined.

Consider now $K_{a, a}$ which is of type 2, thus, by Propositions 3.6 and 4.2,es $\chi_{\chi^{\prime \prime}}\left(K_{a, a}\right)=\lceil a / 2\rceil \leq s d_{\chi^{\prime \prime}}\left(K_{a, a}\right)$. It holds that $s d_{\chi^{\prime \prime}}\left(K_{1,1}\right)=s d_{\chi^{\prime \prime}}\left(K_{2}\right)=1$ and $s d_{\chi^{\prime \prime}}\left(K_{2,2}\right)=s d_{\chi^{\prime \prime}}\left(C_{4}\right)=2$ (see above). For $a \geq 3$ we will use the notations and the described $(a+1)$-total coloring of $K_{a, a}-E^{\prime}$ of the proof of Proposition 3.6, where $\left|E^{\prime}\right|=l=\lceil a / 2\rceil$. This is a partial total coloring of $\left(K_{a, a}\right)_{E^{\prime}}$ where only the subdivided edges and the subdivision vertices are still uncolored.

For each edge $e=v_{i} u_{j} \in E^{\prime}$ with subdivision vertex $w_{i}$ different colors are missing at the end-vertices (namely $a+1$ at $v_{i}$ and 1 at $u_{j}$ except if $j=l$ and $a \geq 5$ is odd, in which case $(3 l+2-l \bmod 2) / 2$ is missing) which can be used to color the edges $v_{i} w_{i}$ and $w_{i} u_{j}$, respectively. Note also that $w_{i}$ is neighbored to four elements, that is, if $a \geq 4$ then there is an available color among the $a+1 \geq 5$ colors of the total coloring of $K_{a, a}-E^{\prime}$ which can be used to color $w_{i}$. If $a=3$, then $w_{i}$ can be colored by color 3 . This completes the $(a+1)$-total coloring of $\left(K_{a, a}\right)_{E^{\prime}}$.

Therefore, $\chi^{\prime \prime}\left(\left(K_{a, a}\right)_{E^{\prime}}\right)=a+1$ and $s d_{\chi^{\prime \prime}}\left(K_{a, a}\right) \leq\left|E^{\prime}\right|=\lceil a / 2\rceil$ follows.

\section{Concluding remarks}

The parameters $e s_{\rho}(G)$ and $s d_{\rho}(G)$ were studied in [7,8] especially for the invariants chromatic number $\chi(G)$ and chromatic index $\chi^{\prime}(G)$. It was shown that $e s_{\chi}(G)=s d_{\chi}(G)$ for all graphs except for bipartite graphs $G$ with cycles, for which $e s_{\chi}(G)=$ $|E(G)|$ and $s d_{\chi}(G)=1$ hold. This difference is caused by the fact that the chromatic number of even cycles increases after one edge subdivision. Moreover, $e s_{\chi^{\prime}}(G)=s d_{\chi^{\prime}}(G)$ for all graphs $G$ with $\chi^{\prime}(G)=\Delta(G)+1$. This means that in these cases it does not matter whether edge removals or edge subdivisions will be carried out.

The results from this paper and from [8] imply that there is no such close coincidence for the two parameters with respect to the total chromatic number, at least for type 1 graphs. For example, $e s_{\chi^{\prime \prime}}(G)=s d_{\chi^{\prime \prime}}(G)$ for empty graphs, paths, cycles $C_{3 k+2}$, complete graphs of even order, and complete bipartite graphs $K_{a, a}$ with $a \neq 2$, but $e s_{\chi^{\prime \prime}}(G) \neq s d_{\chi^{\prime \prime}}(G)$ for acyclic graphs with maximum degree at least 3 , the type 1 cycles $C_{3 k}$ and the type 2 cycles $C_{3 k+1}$ including $K_{2,2} \cong C_{4}$, wheels $W_{n}$ with $n \geq 4$, complete graphs $K_{n}$ of odd order $n \geq 3$, complete bipartite graphs $K_{a, b}$ with $a<b$ except $K_{1,2} \cong P_{3}$, and generalized $\theta$-graphs consisting of at least 3 paths. Moreover, these examples show that the difference between $e s_{\chi^{\prime \prime}}(G)$ and $s d_{\chi^{\prime \prime}}(G)$ can be arbitrarily large for type 1 graphs $G$.

We proved that in general $e s_{\chi^{\prime \prime}}(G) \leq s d_{\chi^{\prime \prime}}(G)$ for type 2 graphs $G$. Equality holds for the type 2 graphs $K_{2 k}$ and $K_{a, a}$ of maximum degree at least 3 which were considered above. Does this hold in general?

\section{References}

[1] S. Akbari, S. Klavžar, N. Movarraei, M. Nahvi, Nordhaus-Gaddum and other bounds for the chromatic edge-stability number, European J. Combin. 84 (2020) \#103042.

[2] D. Bauer, F. Harary, J. Nieminen, C. L. Suffel, Domination alteration sets in graphs, Discrete Math. 47 (1983) $153-161$.

[3] B. Brešar, S. Klavžar, N. Movarraei, Critical graphs for the chromatic edge-stability number, Discrete Math. 343 (2020) \#111845.

[4] G. Chartrand, P. Zhang, Chromatic Graph Theory, Chapman \& Hall/CRC, Boca Raton, 2009.

[5] A. J.W. Hilton, A total-chromatic number analogue of Plantholt's theorem, Discrete Math. 79 (1990) 169-175.

[6] A. J. W. Hilton, The total chromatic number of nearly complete bipartite graphs, J. Combin. Theory Ser. B 52 (1991) 9-19.

[7] A. Kemnitz, M. Marangio, On the $\rho$-edge stability number of graphs, Discuss. Math. Graph Theory 42 (2022) 249-262.

[8] A. Kemnitz, M. Marangio, On the $\rho$-subdivision number of graphs, Discuss. Math. Graph Theory, DOI: 10.7151/dmgt.2412, In press.

[9] A. Kemnitz, M. Marangio, N. Movarraei, On the chromatic edge stability number of graphs, Graphs Combin. 34 (2018) $1539-1551$.

[10] W. Staton, Edge deletions and the chromatic number, Ars Combin. 10 (1980) 103-106.

[11] H. P. Yap, Total Colourings of Graphs, Springer, Berlin, 1996. 